\title{
EchoGéo
}

\section{Juifs d'Istanbul, territorialités d'une communauté entre recompositions et dislocations}

\section{Yoann Morvan}

\section{(2) OpenEdition}

\section{Journals}

Édition électronique

URL : https://journals.openedition.org/echogeo/12419

DOI : $10.4000 /$ echogeo. 12419

ISSN : 1963-1197

\section{Éditeur}

Pôle de recherche pour l'organisation et la diffusion de l'information géographique (CNRS UMR 8586)

Référence électronique

Yoann Morvan, « Juifs d'Istanbul, territorialités d'une communauté entre recompositions et dislocations », EchoGéo [En ligne], 16 | 2011, mis en ligne le 04 juillet 2011, consulté le 25 août 2021. URL : http://journals.openedition.org/echogeo/12419; DOI : https://doi.org/10.4000/echogeo.12419

Ce document a été généré automatiquement le 25 août 2021.

EchoGéo est mis à disposition selon les termes de la licence Creative Commons Attribution - Pas d'Utilisation Commerciale - Pas de Modification 4.0 International (CC BY-NC-ND) 


\title{
Juifs d'Istanbul, territorialités d'une communauté entre recompositions et dislocations
}

\author{
Yoann Morvan
}

\section{Introduction}

1 Sous les feux des projecteurs médiatiques à chaque nouvelle tension entre la Turquie et l'Etat hébreu ou lors des attentats qui ont durement frappé leurs synagogues, les Juifs d'Istanbul constituent la communauté juive numériquement la plus importante en terres d'Islam, avec ceux de Téhéran. En effet, subsiste une communauté d'environ 17000 membres, chiffre estimé, sujet à caution et au cœur d'enjeux symboliques (Bali, 009). Relativement active, cette communauté n'en a pas moins connu d'importants bouleversements au cours du $\mathrm{XX}^{\mathrm{e}}$ siècle.

2 Aujourd'hui, les Juifs d'Istanbul vivent assez dispersés au sein d'une agglomération de plus de 12 millions d'habitants en constante mutation. Les quartiers qui leur étaient traditionnellement dévolus ont été abandonnés au profit d'un certain nombre de secteurs urbains successifs (Kastoryano, 1992b), la communauté s'égrenant ainsi au fil des principaux axes de développement urbain. Cet article en retrace le parcours pour en analyser les enjeux tant urbains qu'identitaires. L'enquête s'est déroulée dans un premier temps à partir des synagogues et de leurs usages, puis par une observation participante jusque dans les foyers de membres de la communauté. La localisation des synagogues reflète l'évolution de la distribution socio-spatiale des Juifs d'Istanbul (Öktem, 2008), jusqu'à un certain point seulement toutefois en raison d'une part des conséquences d'une sécularisation choisie ou subie, et d'autre part des difficultés juridico-politiques à édifier de nouveaux lieux de culte non-musulmans. Dans ce contexte et trois ans après les attentats qui ont touché les synagogues de şişli et de Galata, l'inauguration en 2006 d'une nouvelle synagogue dans l'edge city de GöktürkKemerburgaz, dans l'arrondissement d'Eyüp qui est l'un des fiefs de l'AKP, parti 
conservateur issu de la mouvance de l'Islam politique, a de quoi susciter de multiples interrogations concernant le présent ainsi que le devenir des Juifs à Istanbul.

\section{Tableau équivoque d'une communauté dans l'incertitude}

3 La situation de la minorité juive est pour le moins équivoque dans la mesure où les mêmes faits peuvent donner lieu à des jugements diamétralement opposés. Le bonheur apparent affiché traduit les efforts effectués à visée interne (existentielle) et externe (politique) pour tenter de masquer les contradictions d'une communauté sur laquelle pèsent de nombreuses menaces.

\section{« Tout va très bien Madame la Marquise »}

4 Lorsque l'on interroge la plupart des Juifs d'Istanbul sur le bien-être de leur communauté, leurs réponses commencent quasi-systématiquement par un petit couplet sur le caractère historique de leur présence dans l'ancienne capitale de l'Empire ottoman, sur leur réussite au sein de la mégapole du Bosphore, ainsi que sur leur protection par l'Etat turc. Passé ce discours, non dénué de fondements mais par trop lénifiant, débute la longue liste des motifs d'inquiétude, avec pour finir la question retournée « et en France ...? », sous entendant, comme pour se rassurer, que la vague d'actes antisémites de ces dernières années y serait plus préoccupante qu'en Turquie. Cette séquence en deux temps ressemble fort à la ritournelle "Tout va très bien Madame la Marquise ", chanson phare des années 1930 mise en musique par Ray Ventura, avec des paroles de Paul Misraki (d'ailleurs originaire de Constantinople), deux Juifs français qui durent s'exiler durant la Seconde Guerre mondiale. Néanmoins, l'avenir de la communauté juive d'Istanbul ne paraît pas aussi sombre que la fin de la chanson (château brûlé par un Marquis suicidé parce que ruiné). En effet, elle est dotée d'une infrastructure institutionnelle conséquente, plus ou moins garante d'une continuité dans le temps et l'espace urbain.

5 Le nombre impressionnant, pour une communauté de cette envergure, de synagogues encore actives ou plus généralement d'institutions à but culturel, sportif, ou de bienfaisance, laisse penser que la minorité se porte plutôt bien voire connaît un regain de dynamisme (Bali, 2009). Un autre indicateur peut également consister en une recension des publicités parsemant le journal hebdomadaire communautaire şalom car elles révèlent les aspirations et/ou les territorialités d'une proportion importante de Juifs d'Istanbul. Ainsi, la consultation du numéro précédant la fête juive de nouvel an (en septembre), l'un des plus lus et comportant le plus d'annonces, constitue une sonde probante. On y trouve de nombreuses réclames pour les restaurants de poissons, dont les Juifs d'Istanbul sont très friands; une publicité pour un magasin de vêtement au chic décontracté, situé à Göktürk; des annonces pour des hôtels aux horizons balnéaires, promettant des vacances confortables, ou stambouliotes, certains proposant des salles de réception et repas cacher pour événements religieux familiaux, tel l'hôtel Elite World à Taksim; une offre pour un programme immobilier de prestige, comme dans bon nombre de journaux turcs, mais avec un positionnement plus spécifié, parce que l'entreprise fait partie d'un groupe dirigé par un homme d'affaires juif d'Istanbul, Profilo, et parce que le secteur est déjà investi par des membres de la communauté, la 
péninsule de Bodrum; enfin, des vœux de bonne année exprimés par le maire de l'arrondissement de şişli, où résident toujours de nombreux Juifs. A travers cet inventaire transparaît une certaine image de félicité peu ou prou dissimulée, celle d'une bourgeoisie assez opulente ne boudant pas son plaisir de vivre dans une métropole au charme occidentalisé.

Cependant, tous les membres de la communauté ne partagent pas ce mode de vie puisque, selonses responsables, environ un cinquième de la population juive éprouve de grandes difficultés matérielles. Après plusieurs décennies d'ascension sociale, les classes moyennes connaissent quant à elles un tassement, voire un déclin économique relatif à partir des années 2000. Cela a amené une partie d'entre elles à vendre ou à louer leur résidence secondaire sur les Iles au Prince, lieu privilégié des étés pour les Juifs stambouliotes au $\mathrm{XX}^{\mathrm{e}}$ siècle ${ }^{1}$ jusqu'à ce que: les enfants grandissent et s'y ennuient ; la mode de Bodrum ne la supplante comme destination estivale; le tourisme de plus en plus massif, surtout dans l'île la plus grande (Büyükada), ne vienne troubler l'atmosphère, parfois estimée étouffante, d'entre-soi. Le début de la fin de cette "culture des îles $\|^{2}$ marque un tournant dans les sociabilités juives stambouliotes, altérant cet espace-temps de vivre ensemble propice à la reproduction sociale et identitaire. La plus grande publicisation des espaces publics insulaires vient s'opposer à la devise «pour vivre heureux vivons cachés » que les Juifs d'Istanbul ont faite leur pendant longtemps (Kastoryano, 1992a), devise qui reste de mise aujourd'hui encore, malgré certains accommodements. En effet, les attentats perpétrés contre les synagogues ne sont que la face visible de menaces répétées au cours des deux dernières décennies, contraignant, d'après l'un de ses responsables, la communauté à allouer 10 à $15 \%$ de ses dépenses à sa sécurisation. Toutefois, celles-ci ne couvrent pas les attaques personnelles, comme celle qui a coûté la vie au dentiste Yahia Yasef en août 2003 ou qui a failli faire perdre la sienne en 1993 à l'homme d'affaires Jak Kamhi (patron du groupe Profilo); quant à l'assassinat en 2001 d'Üzeyir Garih (l'un des deux fondateurs du conglomérat Alarko), qui faisait pourtant montre d'un bel optimisme une dizaine d'années plus tôt concernant l'intégration des Juifs de Turquie (şeni, 1990), ses mobiles n'ont pas été clairement élucidés. Ce n'est donc pas un hasard si le dernier venu dans le monde assez restreint des grandes entreprises dirigées par des membres de la communauté est le groupe Pronet, spécialisé dans les dispositifs sécuritaires ${ }^{3}$ : tout autant que les délicieuses bourekas au fromage ou aux épinards, la sécurité devient une spécialité juive d'Istanbul.

Illustration 1 - La synagogue Neve Shalom sous la double protection de Pronet et de la police

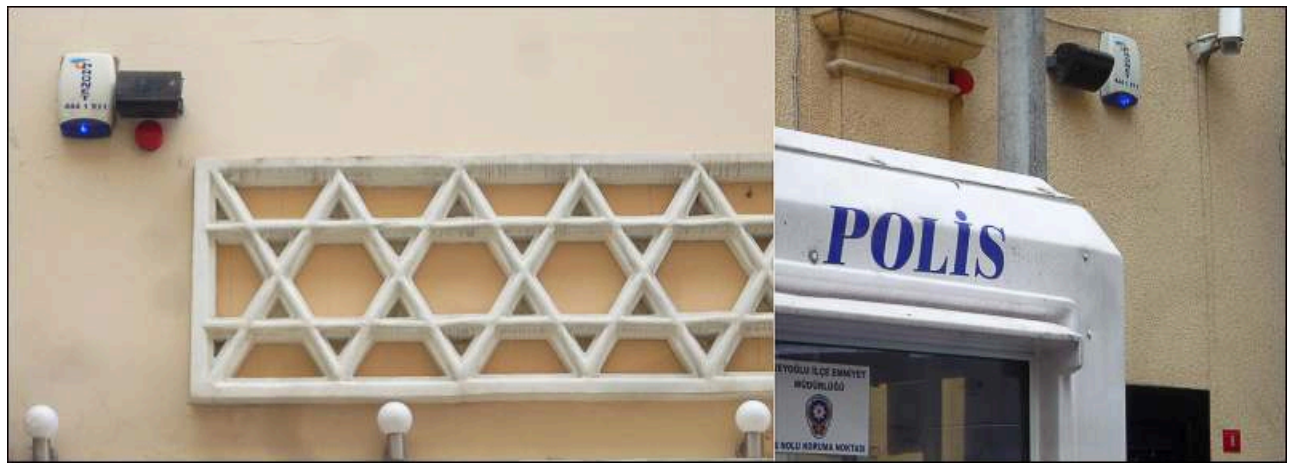

Auteur : Yoann Morvan, mars 2011 
7 Ancienne école réaménagée en synagogue au début des années 1950, alors que le quartier de Galata constituait à cette époque le cœur de la vie juive, Neve Shalom, littéralement "Oasis de paix », porte assez mal son nom. Cible dès 1969, mais sans que cela fasse alors de victimes, elle l'est à nouveau en 1986 (25 morts par balles et par bombes en deux minutes), puis en 1992 (attaque déjouée par les personnels de sécurité), et enfin en 2003 , de manière simultanée avec celle de şişli (250 kg de dynamite tuant 24 personnes dont 18 musulmans au cours de ces 2 attaques) (Güleryüz, 2008). "De paix ", sans doute pas donc, mais Neve Shalom est en revanche une oasis aux allures de bunker où, à défaut de prières quotidiennes depuis le départ des Juifs de ce secteur urbain, se déroulent de nombreux événements communautaires: cérémonies d'investiture des Grands Rabbins (1953, 1961, 2002, 2010), bar mistva, mariages, etc. Les tenues vestimentaires de circonstance, en général "un brin m'as-tu$\mathrm{vu}^{\prime}$, des personnes s'y rendant tranchent bien souvent avec celles des résidents ou travailleurs de conditions plus modestes des rues adjacentes. Discrétion et sécurité obligent, l'entrée s'effectue par une ruelle étroite jouxtant l'ensemble immobilier, puis par un système de sas et de contrôle que l'anthropologue Marcy Brink-Danan compare à ceux pour embarquer pour un vol international dans les aéroports (Brink Danan, 2005). Cette comparaison pose la question du statut actuel des Juifs dans la société et la nation turque. Spécialiste du sujet, l'historien et éditeur Rifat Bali affirme sans concession et avec un pessimisme justifié que ceux-ci mènent une "vie tronquée et à bien des égards au conditionnel»(Bali, 2009). Comment cette extraterritorialité partielle se manifeste-t-elle?

\section{Kemerburgaz versus Kuzguncuk, des territorialités contradictoires ?}

8 Après s'être regroupés au sein de quartiers (en turc "mahalle») où les anciennes minorités ottomanes (Grecs, Arméniens, Juifs) étaient majoritaires, puis s'être polarisés dans des secteurs urbains devenus centraux durant quelques générations (Galata, puis, dans une moindre mesure, şişli, et les Iles au Prince l'été) (Kastoryano, 1992a, 1992b), les Juifs d'Istanbul sont à présent plus disséminés que jamais. Compte tenu de la configuration du site de l'agglomération et de l'historicité de ses établissements humains, la communauté avait certes toujours été spatialement éclatée, mais de nos jours cette absence de centralité propre s'inscrit dans un autre Istanbul que celui de l'Empire ottoman ou des débuts de la République turque : un Istanbul de l'étalement urbain « fractalisé » et de la fragmentation socio-spatiale. Les Juifs participent de cette complexification, avec cependant leurs critères et contraintes spécifiques. Le portrait croisé des territorialités juives de deux contextes urbains très différents en donne un aperçu.

De prime abord, tout semble opposer l'ancien village de Kuzguncuk situé sur la rive asiatique du Bosphore à la très récente et américanisée edge city de GöktürkKemerburgaz implantée au cœur de la forêt de Belgrade. Alors que le premier est un quartier historique où les Juifs vivent depuis plusieurs siècles, comptant plusieurs centaines de familles dans les années 1920, mais dorénavant seulement quelques personnes âgées et pauvres, le second paraît connaitre un destin inverse sur une durée bien plus courte. Les riches «pionniers» installés au début des années 1990 dans le prestigieux complexe résidentiel fermé (en turc «site») Kemercountry se sont aujourd'hui vus rejoindre par 300 à 400 ménages moins fortunés, principalement de 
jeunes couples placés là par leurs familles désireuses de réaliser un bon investissement, dans d'autres site aux fards moins fastes. Soulignant l'importance de l'imbroglio juridico-politique autour du statut de l'enclave de Göktürk ainsi que le caractère décisif de l'ouverture du second périphérique (TEM) dans sa mise en accessibilité, le géographe Jean-François Pérouse a pointé l'effet socio-économiquement moteur exercé par Kemercountry dans l'urbanisation de l'ensemble de la zone (Pérouse, 2004). Parmi les premiers à avoir contribué à lancer cette mode de Kemercountry figurent quelques grandes familles juives, cadres de haut rang dans des grands groupes ou entrepreneurs, en particulier dans l'exportation de textile, les uns et les autres en général venus de Taksim ou de şişli/Nişantaşı et conservant un pied à terre dans l'ancien centre d'Istanbul et/ou ailleurs dans l'agglomération. L'idée de Kemercountry est, selon un de ces Juifs, de recréer l'atmosphère conviviale et élitaire de Burgazada, historiquement considérée plus happy few que celle de Büyükada ${ }^{4}$. Ces "mirages de la distinction " n'hésitent pas à puiser dans l'imaginaire architectural et social stambouliote, y compris celui de Kuzguncuk, pour faire acheter du voisinage trié sur le volet (Pérouse 2004). Paradoxe des urbanités locales, le quartier des bords du Bosphore est désormais en cours de "gentrification", alors que les Juifs y ont laissé leurs maisons, à présent renchéries, il y a à peine quelques décennies.

10 La nostalgie d'un Istanbul révolu amène des pans entiers de la communauté juive à parcourir en automobile de nombreux kilomètres. C'est ce lien entre consommation et nostalgie, réelle ou fantasmée (Appadurai 2005), qui éclaire les territorialités juives relatives à ces deux entités urbaines. Tandis que les Juifs de Kemerburgaz connaissent une mobilité pendulaire pour regagner leur entre-soi aux connotations parfois nostalgiques, ceux originaires de Kuzguncuk y reviennent de façon hebdomadaire pour faire revivre la synagogue et ses alentours le temps d'un shabbat ou d'une fête. L'attachement à ce quartier est si puissant qu'il entraîne même des discordances entre les géographies cultuelle et existentielle dans la mesure où bon nombre des personnes y commémorant les offices religieux habitent non loin d'autres synagogues autrement plus insérées dans des territorialités juives actuelles : şişli et surtout Caddebostan. Leur attachement s'enracine dans leurs souvenirs de vie juive déployée dans les espaces publics du quartier, devenues rues de la mémoire (Mills 2010). Majoritairement musulman depuis le courant des années 1950, puis "gentrifié » à partir de 1980 et plus encore 2000, Kuzguncuk respire encore aujourd'hui cette tranquillité, les Juifs qui s'y rendent pouvant davantage qu'ailleurs stationner par petits groupes aux alentours de la synagogue sans se sentir en danger ou se faire disperser par les forces de sécurité. Il s'agit là d'un autre point commun avec Kemerburgaz. Il est ainsi remarquable que les deux synagogues de ces secteurs urbains pourtant si différents soient sans doute les moins sécurisées de toutes celles d'Istanbul. La synagogue de Göktürk est très discrètement située au dernier étage d'un immeuble dont les autres occupants en disent long sur les styles de vie des résidents de cette entité urbaine ${ }^{5}$. Moins aseptisés et globalisés, les commerces environnant celle de Kuzguncuk, qui en revanche a pignon sur rue, sont d'un standing comparable. La divergence la plus manifeste entre les deux cas est en définitive leur relation à l'exil. Alors que les résidents de Göktürk ont fait le choix d'une forme d'exil intérieur, substitut d'alya vers Israël ou d'émigration aux Etats-Unis, ceux qui ont grandi à Kuzguncuk vivent leur éloignement de leur quartier natal sur un mode plus défectif que seul le retour périodique vient compenser ${ }^{6}$. 


\section{Retour sur un siècle de trajectoires juives}

11 En un siècle, Istanbul a assisté à des changements considérables. Après la perte de son statut de capitale et le départ forcé de la majorité de ses minorités, synonymes de stagnation et de turquification, un boom démographique sans précédent a fait passer la population d'un million vers 1950 à 7 millions en 1990 et plus de 12 millions aujourd'hui ; ses horizons urbains se sont démultipliés, le territoire d'Istanbul croissant de 2000 à 500000 hectares, les nouveaux secteurs urbanisés fleurissant tous azimuts (Pérouse, 2010). Ce siècle stambouliote (1910-2010), les Juifs l'ont traversé en plusieurs sens du terme. En premier lieu, cela porte l'accent sur les épreuves récurrentes qui ont jalonnées leur présence en Turquie (évictions forcées de Thrace en 1934, impôt inique en 1942, vandalismes anti-minoritaires en 1955, etc.), épreuves ou événements, tel l'indépendance d'Israël en 1948, les encourageant à l'émigration ou à de profondes transformations identitaires. En un sens bien moins tragique, la traversée est aussi celle d'une rive à l'autre du Bosphore, favorisée par la construction de deux ponts (1973 puis 1988). En effet, hormis quelques exemples notoires d'établissements juifs à l'année (Kuzguncuk à Üsküdar, Haydarpaşa à Kadıköy), la partie asiatique d'Istanbul était considérée avant tout comme un lieu de villégiature (Akbulut 2010). A présent, l'axe articulé autour de Bağdat Caddesi («l'avenue de Bagdad») constitue l'un des foyers majeurs du judaïsme stambouliote avec plus de 1500 familles installées de Kadıköy à Pendik (Güleryüz, 2008), et, pour une part en raison de leurs trajectoires, c'est la composante la plus homogène, religieusement et socio-culturellement, de la communauté.

\section{Mobilités résidentielles et linguistiques}

12 Si autrefois les incendies étaient le facteur prépondérant de la mobilité résidentielle, fournissant l'occasion de déménager vers des habitats et des quartiers plus modernes, priment depuis lors les effets de mode et d'opportunités qui font de la mégapole un vaste Monopoly grandeur nature. Les choix communautaires demeurent mais tendent à s'atténuer au profit des toutes dernières tendances du moment, comme en témoignent les quelques familles ayant investi les improbables site d'Avrupa Konutları (près du TEM, le second périphérique d'Istanbul) ou les dizaines de ménages dans les immeubles d'Ataşehir (à l'intérieur de la rive anatolienne). Cependant, outre les implantations vectorisées par la Bağdat Caddesi, ce qu'il est convenu d'appeler "l'axe du nord", esquissé dès le XIXe siècle, s'est prolongé, densifié et diffracté à la suite de l'ouverture des deux ponts (Köksal, 2010). Auteure de l'article de référence sur les «trajectoires dans la ville » des Juifs d'Istanbul, Riva Kastoryano emploie un joli jeu de mot turc pour décrire ce "mouvement vers le haut", signifiant ainsi la double ascension sociale et spatiale (Kastoryano 1992b). Le schéma «classique » fait partir les Juifs de Balat ou de Hasköy avec ensuite une importante étape à Galata ; puis arrive le temps de Taksim, suivi de ceux de şişli/Kurtuluş/Nişantaşı en fonction des classes sociales, et enfin Gayrettepe. Une séquence ultérieure les mène sur les hauteurs européennes du Bosphore d'Ulus, d'Etiler et d'Istinye ou la clairière de Kemerburgaz, plus au nord dans la forêt mitée par l'urbanisation. Maslak, notamment l'ancienne coopérative devenue site des Gazeteciler (plusieurs centaines de personnes, semble-t-il), représente la dernière escale à ce jour, bien que les abords de la mer Noire (Zekeryaköy, Kilyos et bientôt peut-être Riva, coté asiatique) ne soient pas en reste. Que de chemin parcouru 
depuis les conversations en ladino aux débuts du siècle dernier sur les berges de la Corne d'Or jusqu'aux écrans plats de télévision retransmettant CNN Türk dans de cossus intérieurs aux esthétiques postmodernes ...

Ce processus de métropolisation n'est toutefois pas aussi linéaire qu'il n'y paraitt. Il faut commencer par souligner qu'il ne concerne pas uniquement les Juifs d'Istanbul mais d'une manière plus générale l'ensemble des Juifs de Turquie (voire d'une partie des Balkans, de la mer Noire ou du Proche-Orient), pour lesquels l'ancienne capitale de l'Empire ottoman est quasiment devenue l'unique point de regroupement dans l'Etat fondé par Atatürk ${ }^{7}$. La liste des communautés établie par Abraham Galante vers le milieu du XX ${ }^{\mathrm{e}}$ siècle a de quoi surprendre aujourd'hui : de Manisa à Van jusque Hakkâri, de Trabzon à Gaziantep, en passant par Diyarbakır, il note une présence juive passée ou contemporaine de son enquête dans un nombre incroyable de villes alors petites ou moyennes (Galante, 1985). Si une bonne partie de ces communautés isolées a migré vers Israël, une proportion non négligeable est venue vers la grande ville. Les Juifs d'Istanbul sont trop souvent présentés comme étant sépharades "à $95 \%$ " et ashkénazes pour le reste, pourtant une assez grande diversité d'origines, notamment linguistiques, caractérise le paysage communautaire. En particulier, les originaires de l'ancien arrière-pays d'Alep (Urfa, Gaziantep, Hatay), initialement arabophones, les Géorgiens arrivés il y a environ un siècle ou ayant transité par l'est de la Turquie, ou encore les Juifs kurdes constituent autant de populations non sépharades au sens strict (descendants de Juifs ayant fui l'Espagne du fait de l'Inquisition); certaines de ces origines sont repérables à travers les noms de famille, bien que la traçabilité perde au fil des générations de sa fiabilité. Les premières concernées par la migration vers la métropole ont été les communautés des alentours de la mer de Marmara (Bursa, Çanakkale), ainsi que celles de Thrace mais pour des raisons politiques nationales.

Illustration 2 - Carte des villes et régions citées en Turquie et dans les pays limitrophes

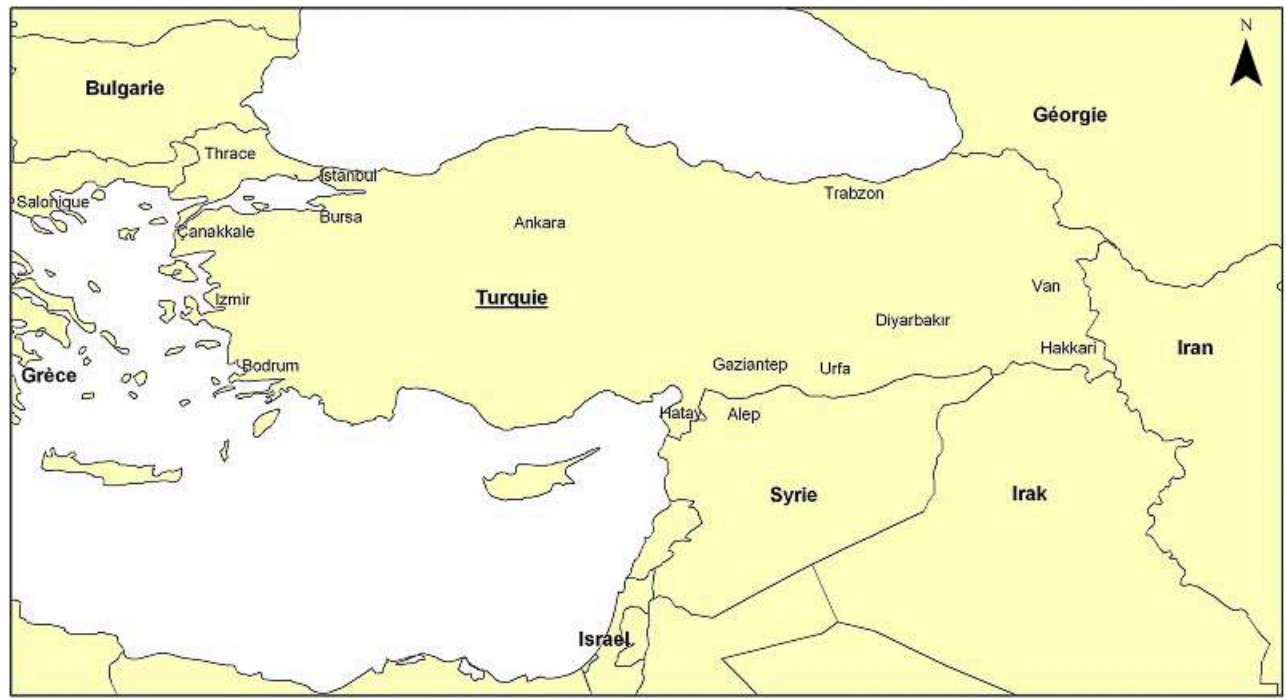

II s'agit d'une carte simplifiée indiquant des lieux qui ont leur pertinence à des époques différentes, d'où certaines incongruités, telles l'absence de limites à la Géorgie (qui n'est devenue une entité nationale que récemment) ou la présence de Bodrum aux cotés de villes d'une toute autre consistance historique.

Auteur : Yoann Morvan, mars 2011. 
Illustration 3 - Istanbul : la mégapole stambouliote en expansion

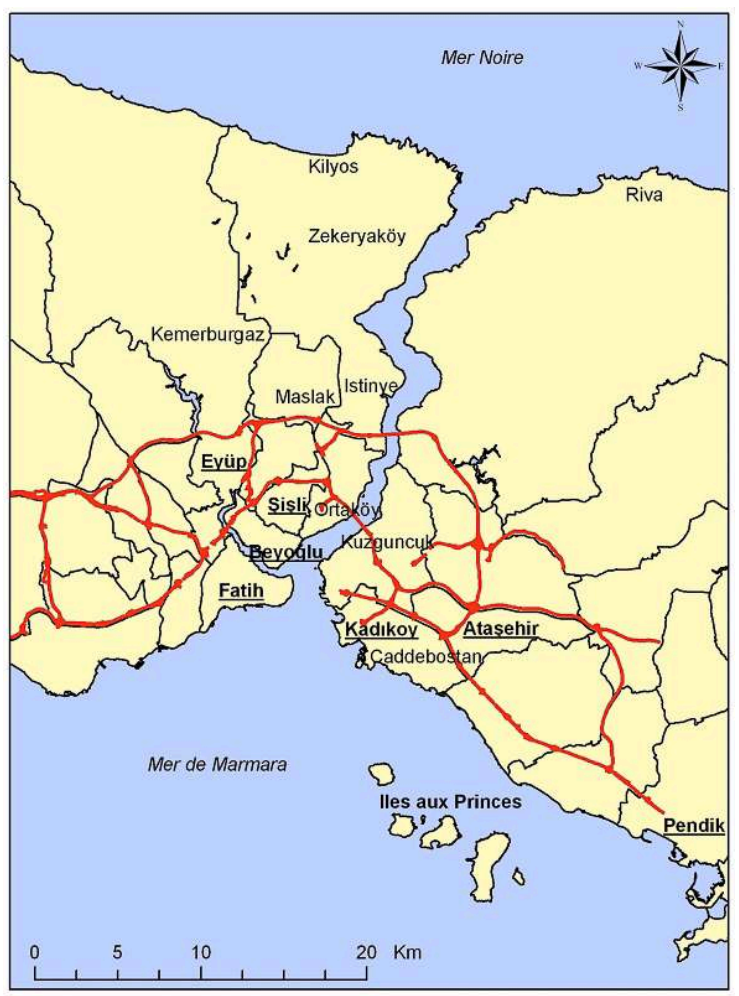

En rouge, les autoroutes urbaines ; en noir, les limites d'arrondissement du Grand Istanbul ; souligné, les noms des arrondissements cités.

Auteur : Yoann Morvan, mars 2017. 


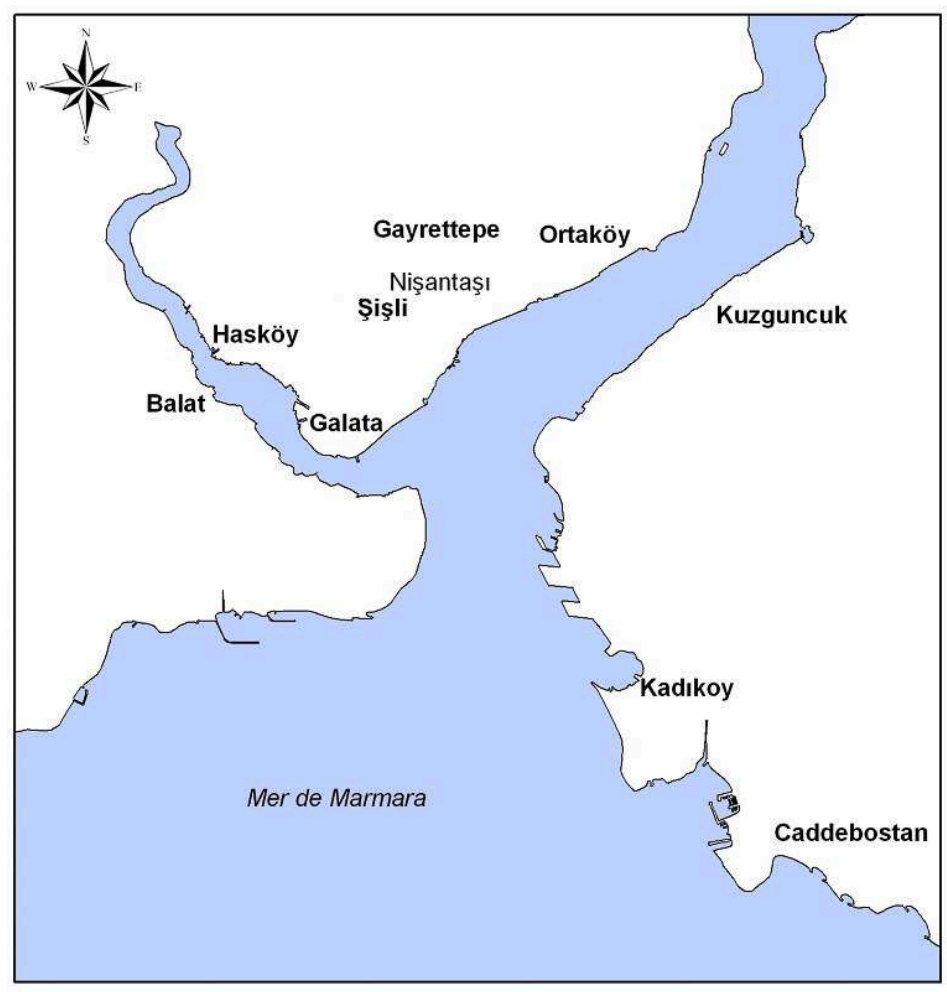

Auteur : Yoann Morvan, mars 2011

Pour revenir aux migrations intra Istanbul, les points nodaux, via lesquels les populations juives sont ensuite redistribuées, sont au premier chef Galata, puis şişli. Reprenant à juste titre le modèle d'« écologie urbaine » conçu par l'Ecole de Chicago, Riva Kastoryano décrit le mécanisme de "push and pull» interne et externe à la communauté juive: chaque nouvelle arrivée de populations plus pauvres, juives ou turques d'Anatolie, faisant fuir celles plus prospères, installées antérieurement, vers des secteurs urbains jugés plus modernes, et Galata faisant figure de «stade de transition", selon l'expression de Louis Wirth, entre tradition et modernité dans ce mouvement évolutif (Kastoryano, 1992b). Ce statut intermédiaire des environs de la tour de Galata se retrouve dans sa toponymie juive, «la Kula », hispanisation du turc «kule» ("tour»), ultime espace de pratique traditionnelle du ladino à l'échelle d'un quartier, notamment parce que ses derniers habitants juifs dans les décennies 1950-1970 étaient de conditions assez modestes. Inversement, Galata a constitué le premier accès aux immeubles à appartements, synonymes de modernité et instaurateurs de fines distinctions sociales d'une rue ou d'un bâtiment à l'autre. Cette forme urbaine sera la norme jusque Gayrettepe coté européen et Bostanc1 coté asiatique. Débute ensuite une séquence d'histoire sociale des mobilités résidentielles dont la théorie des mouvements browniens est plus à même de rendre compte que celle de l'Ecole de Chicago. Les disparates complexes résidentiels fermés et leurs accès autoroutiers se voient préférés aux immeubles et à l'ancien réseau viaire dans lesquels les moins aisés se trouvent en quelque sorte stockés, miroir aux alouettes d'une modernisation et d'une mobilité sociale ascendante bloquées : jouxtant şişli, le quartier de Kurtuluş (mot qui signifie en turc "libération", ce que le quartier a socialement représenté pendant une courte période) en fournit un bon exemple de nos jours, et 
Nişantaşı ou Caddebostan des contre-modèles. En dépit du caractère relativement épiphénoménal de certaines concentrations par immeuble ou site, persiste affectivement l'image de " quartiers juifs ». Cependant, sauf exceptions notables tels les contreforts récemment construits d'Ortaköy vers Ulus (significativement, en turc le mot désigne la "nation») avec un Carrefour a leur pied vendant depuis peu des produits cachers, la notion se vide progressivement de son sens.

A ces trajectoires résidentielles correspondent des évolutions linguistiques, et cette correspondance symbolise bien davantage qu'une concomitance fortuite. En effet, nous habitons les langues autant qu'elles nous abritent et nous meuvent, maisons et ressources tout à la fois. Outre la diversité linguistique liée aux origines (dans des proportions très variables donc : ladino, italien, allemand, yiddish, russe, arabe, kurde, géorgien), les Juifs d'Istanbul se sont longtemps distingués par leur polyglottisme acquis au bénéfice de leurs voisinages et activités professionnelles (turc, grec, arménien) ou de leurs apprentissages (hébreu, français, anglais). L’âge d'or de ce polyglottisme est le demi-siècle allant de 1910-1920 à 1950-1960. Des routes commerciales des villes anatoliennes au lycée francophone Notre Dame de Sion ou des intérieurs londoniens aux rues cosmopolites de Péra, le roman fleuve de Mario Levi, Istanbul était un conte, en décrit avec justesse les territorialités (Levi, 2010). Cette période est aussi celle de l'effacement du ladino, langue maternelle de $81 \%$ des Juifs d'Istanbul en 1945 contre seulement $33 \%$ en 1965. Sans disparaitre totalement malgré le départ, principalement en Israël, d'un grand nombre de ses locuteurs, il s'étiole sous les coups de boutoir du français puis du turc, ainsi qu'au gré des mutations sociourbaines de la communauté, comme l'a bien montré le linguiste Reynaldo Romero (2008). Le ladino n'est pas la seule langue juive d'Istanbul à connaître un tel destin puisque l'on assiste en fait à une réduction unificatrice de la diversité linguistique et alphabétique au profit d'un turc épuré et écrit en lettres latines. Cette transformation va de pair avec la laïcisation d'une communauté juive turque pendant longtemps assez peu nostalgique du ladino considéré comme un signe d'arriération, notamment religieuse, et de moins en moins en mesure de déchiffrer l'alphabet hébraïque, danger déjà pointé en son temps par le pourtant très kémaliste Galante (1985). La phonétisation du turc et par ricochet de l'hébreu coïncide avec une standardisation éparpillée de l'habiter. De façon plus préjudiciable, tandis que la mobilité résidentielle se complexifie et s'accroît, celle permise par les langues, tendant à se limiter au turc et à l'anglais, se restreint et se banalise.

\section{Parcours identitaires}

16 La relation des Juifs d'Istanbul aux langues qui ont été ou qui demeurent les leur est un excellent fil d'Ariane pour retracer leurs parcours identitaires. Comme les langues, les identités ne sont pas des données fixes, s'adaptant de manière diachronique aux contextes successifs. La honte du ladino et de l' « accent juif » en turc va inciter les Juifs d'Istanbul, désireux d'apparaitre moderne, à les abandonner ou les occulter pour lui préférer le français, symbole de distinction sociale, la turquification venant quant à elle s'ériger contre ces deux langues. Pour une partie de la communauté, ces processus contradictoires ont provisoirement et paradoxalement produit certaines formes d'enrichissement car ils ont contraint les Juifs à augmenter leurs ressources linguistiques et à renforcer leur statut économique d'intermédiaire avec l'international. En revanche, une fois ce processus de turquification quasiment achevé, 
les Juifs d'Istanbul vont perdre cet avantage comparatif, la jeunesse turque maîtrisant de mieux en mieux les langues étrangères; l'égalisation depuis les années 1980 des écarts financiers entre les hommes d'affaires juifs et musulmans constitue notamment l'aboutissement de politiques de la République turque de spoliation progressive de la minorité juive, dont l'impôt sur la fortune de 1942 représente l'acmé (Bali, 2009). Ainsi, malgré les efforts consentis, le bilan de ce parcours identitaire est assez largement négatif.

$17 \mathrm{Au}$ moment où s'est instituée la République, espérant à tort « jouir pleinement de leur citoyenneté turque sans avoir à renoncer à leur identité ", les Juifs de Turquie se sont trouvés confrontés à un "dilemme identitaire », ambivalences dont Rıfat Bali a bien retracé les linéaments, (Bali, 2010). Il s'en est suivi une turquisation des noms de famille ${ }^{8}$ et des prénoms, ainsi que d'une partie de la liturgie. Sur ce dernier point, de fines différences existent d'une synagogue à l'autre, celle où le ladino est le plus utilisé étant celle de Kuzguncuk. Encore une fois, l'opposition avec Kemerburgaz est moins patente qu'il n'y parait, l'une des personnes prônant le plus ardemment la défense du ladino a choisi de résider à Kemerburgaz. Réduite à sa portion congrue dans les pratiques quotidiennes, cette langue fait surtout aujourd'hui l'objet d'un usage fragmentaire mais ô combien révélateur, en particulier le terme « vedre », " vert », pour désigner les musulmans sans que ces derniers ne soient capables de le comprendre. L'un des analyseurs de la relation au ladino et plus généralement de l'évolution identitaire des Juifs d'Istanbul est l'organe communautaire şalom, dernier représentant d'une presse juive bien plus nombreuse jusqu'aux années 1930. Son premier numéro sort le 29 octobre 1947, date anniversaire de la création de la République turque, et après un succès relatif à ses débuts, le journal connait un déclin, lié à celui du ladino, que la reprise par une nouvelle équipe rédactionnelle vient stopper en 1984, faisant de şalom, fort de ses 3000 abonnés, "l'instrument de survie» des horizons identitaires judéo-espagnols et judéo-turcs (Marcilhacy, 2005). L'hebdomadaire comporte de nos jours une page en ladino et publie un supplément mensuel «El amaneser » dans cette langue, les articles y oscillant entre folklorisation et, comme en témoignent les doctes écrits de Moşe Palaçi en matière religieuse, des tentatives de continuation du mince filet des traditions. Si la dislocation, identitaire comme urbaine, n'est pas très loin, de timides forces de recompositions se font jour.

Presque invisibles à l'échelle de l'espace social global d'Istanbul, de nouveaux types de regroupement s'opèrent à la faveur d'un « effet club " qui ne se confond pas totalement avec le communautarisme. Evoquées précédemment, les « gated commnunities alaturka » sont massivement investies par les classes moyennes et supérieures, notamment celles de la communauté juive. Outre les exemples déjà mentionnés, le cas de la site Alkent à Etiler étudié par Ségolène Debarre est assez paradigmatique: les familles juives $\mathrm{y}$ seraient une centaine environ, attirées par l'appartenance à la communauté du promoteur et le bouche à oreille, forte minorité donc mais sans que ce réseau soit prépondérant, d'autres effets clubs influençant tout autant son peuplement et ses sociabilités, tel celui des anciens élèves du lycée Galatasaray, ces différents réseaux rentrant parfois dans des logiques oppositionnelles (Debarre, 2004). L'effet club joue ainsi à des niveaux imbriqués, au sein des complexes résidentiels fermés et parfois entre eux, comme l'a montré une expérience unique qui s'est déroulée durant les travaux de sécurisation de la synagogue d'Ortaköy suite aux attentats de 2003. Le lieu de culte a été clos pendant un peu plus d'une année et les offices religieux se sont 
effectués chez les uns et les autres entre Ulus et Etiler, les rouleaux de la Thora passant d'une gated community à une autre semaine après semaine. Cela a contribué à la formation d'une sorte de club des amis de la synagogue d'Ortaköy, auparavant moins fréquentée et par des fideles de conditions plus modestes (moins de 30 personnes vivaient dans ses alentours immédiats au début des années 1990), club trouvant son prolongement dans la proximité spatio-temporelle du House Café des bords du Bosphore. Cette chaîne de cafés colle d'ailleurs au plus près des territorialités juives d'Istanbul : le premier s'est ouvert en 2002 à quelques mètres des locaux de şalom, un deuxième s'installant non loin sur la Teşvikiye (Nişantaşı), les autres se localisant à Tünel et sur Istiklal (principale artère commerciale de Beyoğlu), sur les collines d'Etiler et les rivages de Bebek et de Caddebostan, ou dans les malls d'Istinye Park et de Kanyon. Tous ces lieux sont très prisés par les Juifs et plus généralement, mais pas seulement, par les "Turcs blancs ", espaces de consommation symbolique des lifestyles américanisés. Se calquant sur cet apparaitre, une majorité des Juifs d'Istanbul miment l'assimilation, au risque des mariages mixtes et des entorses aux lois religieuses en particulier pour ceux, en nombre non négligeable, revenant vers leurs pratiques. « Fort comme le fer, fragile comme la rose ", c'est aussi une manière de se protéger par cette stratégie d'invisibilité publique (Neyzi, 2005).

\section{Quels Juifs pour quel Istanbul?}

19 Les territorialités et reterritorialisations des Juifs d'Istanbul doivent être mises en perspectives avec les évolutions démographiques de long terme et les changements politiques récents. En effet, l'entrecroisement, notamment par les jeux d'acteurs qui se nouent au niveau politique, de ces deux données pèse de tout son poids dans l'ambivalente dynamique de dislocation/recomposition. De ce fait, les questionnements autour des patrimoines revêtent ainsi une signification à la fois pragmatique et symbolique de la place occupée aujourd'hui par les Juifs d'Istanbul.

\section{Perspectives politiques et démographiques}

Les interrogations portées quant au devenir de la communauté juive d'Istanbul se font de plus en plus inquiètes. La lente mais inexorable érosion démographique est masquée par les instances rabbiniques comme par les autorités politiques turques actuelles pour des raisons convergentes : assurer que les minorités non musulmanes, juive dans le cas présent, vivent dans un climat de « tolérance et de paix » (Bali, 2009), à l'instar de celui supposé avoir prévalu durant l'époque ottomane. La prétendue stabilité démographique volerait ainsi au secours d'une volonté politique alors que l'estimation la plus basse, autour de 17000 , est elle-même sans doute à l'heure actuelle surévaluée. La diminution du nombre des Juifs en Turquie s'est réalisée par étapes : 82000 en 1927, puis 77000 en 1948, ils ne sont plus que 46000 en 1951, trois ans après la création de l'Etat d'Israël, et passent à 38000 en 1965 (Bali, 2009). Ce déclin doit être mis en perspective avec les évolutions démographiques, selon des chronologies un peu différentes, des anciennes minorités grecque (100 000 en 1927, 47000 en 1965 contre environ 4000 de nos jours), ou arménienne (30 000 rejoints par un nombre comparable de migrants venus d'Arménie récemment), et surtout avec l'accroissement considérable de la population turque à Istanbul comme dans l'ensemble du pays (24 millions en 1955 
et 77 millions aujourd'hui). Le poids relatif des Juifs est donc en chute libre, chute que la natalité ne vient en rien compenser. Seul $20 \%$ des membres de la communauté sont âgés de 0 à 24 ans, ce chiffre est de $50 \%$ à l'échelle nationale (Bali, Mallet, şeni, 2009). Tout aussi préoccupante que cette pyramide des âges vieillissante est la situation des cadres potentiels de la communauté dans les décennies à venir. Un cercle vicieux s'installe dont les strates intermédiaires sont les grandes perdantes, la structure communautaire s'acheminant vers une scission accentuée entre des démunis sans ressources pour migrer et des possédants ne pouvant pas ne pas rester, cela rendant les stratégies matrimoniales assez ardues (Behar, 2006) en raison du rétrécissement de l'espace des choix et du clivage social interne. Les jeunes étudiants en Israël, en Europe et davantage encore aux Etats-Unis ont tendance à ne pas revenir. Une proportion croissante des couples de 35 à 45 ans avec de jeunes enfants et des moyens financiers conséquents, surtout ceux impliqués dans la vie communautaire et éducative, songent de plus en plus à partir, ne voyant pas d'avenir pour leur progéniture en Turquie. Le contexte politique n'y est pas étranger.

21 Les rapports de la communauté juive d'Istanbul avec les autorités ottomanes puis républicaines n'ont jamais été simples, incitant les Juifs à adopter une attitude de profil bas. Au début des années 1990, quelques lueurs d'espoir, dont l'établissement de relations diplomatiques avec l'Etat hébreu, laissaient présager d'une amélioration de leur condition (Mallet, 2008), mais ces espoirs ont été vite déçus. La montée en puissance d'un AKP politiquement de plus en plus hostile à Israël les a même conduits à un nouveau type d'instrumentalisation en matière internationale, rôle qui leur est dévolu en guise d'intégration (Kastoryano, 1992c), bien plus schizophrénique que le lobbying anti-arménien. La communication malaisée de la cellule de crise mise en place par la communauté pendant l'affaire du Mavi Marmara (arraisonné au large de Gaza par l'armée israélienne le 31 mai 2010), implicitement sommée de manifester son antisionisme, en est la preuve la plus éclatante. Comme l'explique Rifat Bali, les dirigeants communautaires doivent, aujourd'hui comme hier, composer avec un «nombre réduit d'options » et une « marge de manœuvre étroite », déplorant que « la marche de la Turquie vers plus de démocratisation et de libéralisation n'ait eu que si peu de conséquences positives à long terme pour la communauté juive du pays » (Bali, 2009). Néanmoins, à mots couverts, certains Juifs d'Istanbul ne cachent pas leur sympathie paradoxale envers le parti islamo-conservateur, satisfaits des résultats obtenus dans le jeu qu'ils sont contraints de jouer et dont ils ne maîtrisent nullement les règles. Un membre de la loge B'nai Brith me confiait ainsi qu'avec l'AKP les réponses aux diverses demandes sont quasi immédiates, efficacité clientéliste qui contribue à séduire des électorats variés. A l'échelon des mairies d'arrondissement, la chose est encore plus flagrante ; toutes les institutions juives présentes dans les arrondissements contrôlés par l'AKP cherchent à nouer de cordiales relations avec le parti d'Erdoğan, liens «bienveillants» que celui-ci utilise en retour comme plus-value en termes d'image de non-antisémitisme. Dans cette perspective, dans l'arrondissement d'Üsküdar la synagogue de Kuzguncuk programme des rencontres interreligieuses, dans celui d'Eyüp l'espace cultuel de Kemerburgaz a pu voir le jour ${ }^{10}$, mais le cas le plus probant est sans doute Beyoğlu. En effet, c'est à la fois l'arrondissement d'Istanbul qui a le plus de visibilité et celui qui compte le plus grand nombre d'institutions juives: hospice, synagogues, grand rabbinat et le musée juif du $500^{\mathrm{e}}$ anniversaire (de l'arrivée des Sépharades fuyant l'Inquisition). Ce dernier est la parfaite illustration de l'idéologie de la tolérance promue, son responsable intitulant significativement son dernier 
ouvrage "Les Juifs turcs, 700 ans d'être-ensemble», dressant un portrait hagiographique de la « bienheureuse » histoire judéo-turque (Güleryüz, 2009). Le partipris muséologique de ce musée inauguré en 2001 dans l'ancienne synagogue Zülfaris révèle l'échange symbolique aux allures néo-ottomanes réalisé via le thème de la tolérance : la demande de reconnaissance, sur le mode du consentement et des faveurs commercées (Brink Danan, 2010). De tels marchandages dans un contexte de déclin démographique augure d'embarras grandissants quant au sort réservé aux patrimoines hérités dont la transmission semble problématique.

\section{Patrimoines juifs d'Istanbul en questions}

La question patrimoniale apparaît assez emblématique de la place occupée par les Juifs en Turquie. Vêtements trop amples pour une communauté réduite, les patrimoines juifs d'Istanbul peuvent être analysés, en complétant cette approche par la dimension politique décrite ci-dessus, à la lumière des travaux de Françoise Choay, pour qui la notion même de patrimoine charrie une " touristification » rampante de notre relation au passé (Choay, 2009). Les manifestations estampillées «Istanbul 2010 Capitale européenne de la culture » participent de cette mise en marque et certaines d'entre elles ont concernées les patrimoines juifs. L'une des plus réussie a été l'installation minimaliste d'art contemporain Molecular dans l'ancienne synagogue de la Mayor, détruite en 1923 par un incendie et occupée depuis par des ateliers d'artisans. Elle se situe à Hasköy à proximité d'une autre synagogue qui après avoir servi de magasin de goudron puis de fonderie, a été cédée par la municipalité de Beyoğlu à un investisseur privé, qui l'a rénovée et transformée en café touristique. Le destin de ces deux édifices cultuels témoigne de la difficulté de la conservation du patrimoine dans une vocation autre que touristique. "Bâti orphelin, malmené », faisant l'objet de "spoliations spontanées », notamment pour faire des parkings, tel le triste exemple de la synagogue Kastorya à Balat de l'autre coté de la Corne d'Or ${ }^{11}$, les biens non-musulmans sont soumis à rude épreuve une fois les territorialités désinvesties, les présences «en creux » (Pérouse, 2005). La question juridique pèse aussi de tout son poids, les autorités turques exerçant un contrôle pointilleux sur l'immobilier des vakıf («fondations ») des anciennes minorités ottomanes. Une des nécessités impérieuses pour la communauté est d'occuper le terrain, symboliquement par des rénovations parfois luxueuses, ou même physiquement, quitte à payer des gens pour cela, en premier lieu des Juifs pauvres. Ce procédé ne peut évidemment pas valoir pour le cas des cimetières, également très soumis à la pression spéculative compte tenu de leur taille et de leur emplacement, celui d'Ortaköy en particulier. Souvent le mal est déjà fait et il ne reste plus qu'à préserver une butte témoin de la ruine, comme le mausolée Camondo bientôt modestement restauré par la communauté juive et Istanbul 2010, Capitale européenne de la culture. 
Illustration 5 - Schneider Tempel, ex synagogue Tofre Begadim

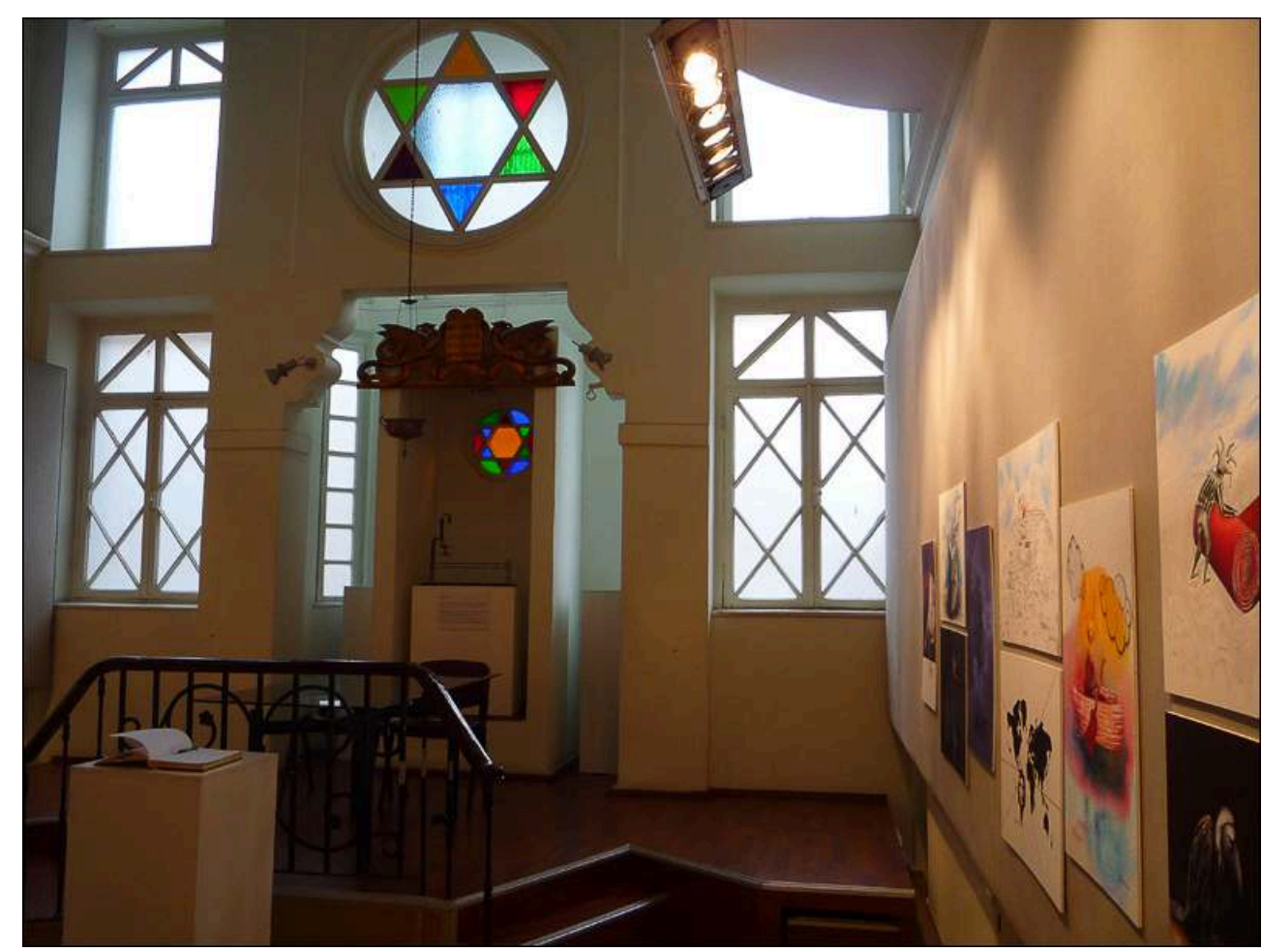

Autrefois dévolue aux classes populaires ashkénazes, cette synagogue est transformée en galerie d'art en 1999.

Source : Yoann Morvan, mars 2011

Un rituel religieux est particulièrement respecté par les Juifs d'Istanbul, la commémoration de la mort d'un proche disparu, rite toujours dénommé en ladino: «meldado ». A l'occasion du décès du défunt, la famille se destitue bien souvent d'une grande part des livres de prières dont elle n'a plus l'usage, ces livres contenant le nom de Dieu étant ensuite enterrés au cimetière. L'impasse de la transmission se donne aussi à lire dans le paysage urbain des hospices juifs dédiés à la solidarité envers les ainés nécessiteux, dont l'un est situé au fond d'un cul-de-sac et l'autre était une ancienne école de l'Alliance Israélite Universelle. A l'autre bout des âges de la vie, la communauté a tenté de freiner l'acculturation, en particulier celle des jeunes des classes sociales aisées, en recréant un lycée juif à Ulus dispensant de 3 à 5 heures d'enseignements en hébreu. Accueillant 600 élèves, son succès est mitigé, certains de ses détracteurs l'accusant d'être un établissement pour les Juifs plus que véritablement judaïque, d'autres estimant son niveau trop faible en comparaison de structures scolaires plus prestigieuses et plus propédeutiques en vue d'accéder aux meilleures universités. En effet, l'un des enjeux pour les familles riches est de former une élite communautaire destinée à prendre la suite de l'entreprise paternelle ou d'intégrer des grandes sociétés. La structure très oligarchique de la communauté et ses effets redistributifs en cascade la rend économiquement assez fragile. Si les puissants en venaient à disparaître, l'ensemble des Juifs d'Istanbul se trouverait de près ou de loin en précarité relative. La question du patrimoine est ainsi également celle de la valeur des biens et des activités. Les investissements réalisés dans les site du nord de l'agglomération, bien que produits immobiliers de courte durée (Pérouse, 2004), devraient bénéficier de l'ouverture des troisièmes pont et périphérique (Morvan, Montabone, 2010). En ce qui concerne les 
activités professionnelles, les transformations des secteurs du commerce et du textile ont conduit notamment les plus âgés à compléter leurs revenus par l'exercice de prêts à court terme très rémunérateurs, mais aux limites de la légalité et hautement périlleux. La dialectique entre de fructueux placements à Istanbul et la rapide remobilisation des capitaux est plus que jamais d'actualité. Lors de la dernière période de congés en Turquie (novembre 2010), environ 2000 Juifs d'Istanbul seraient allés en Israël et une bonne partie d'entre eux y aurait prospecté afin d'acheter un bien immobilier, au cas où ... A Istanbul, l'une des fêtes juives annuelles drainant le plus de fidèles a été organisée en 2010 dans l'un des sous-sols du nouveau quartier d'affaires de Maslak par un émissaire du mouvement charismatique américain 'Habad, l'ambiance fantaisiste et vaguement branchée y est digne d'un night club londonien, elle vient célébrer Pourim, la Fête des Sorts.

\section{BIBLIOGRAPHIE}

Akbulut R., 2010. Kadıköy. L'autre ville de l'autre côté. Urbanisme, $\mathrm{n}^{\circ}$ 374, septembre-octobre 2010, p. 43-45.

Appadurai A., 2005. Après le colonialisme. Les conséquences culturelles de la globalisation. Paris, Payot. Bali R., 2009. Entre nationalisme et islamisme : la lente disparition de la communauté juive de Turquie. In La fin du judaïsme en terres d'Islam, Trigano S. (dir.). Paris, Denoël.

Bali, R., Mallet, L., şeni, N., 2009. Juifs turcs ou juifs de Turquie. Film documentaire, 125 min, Image6 Musée d'art et d'histoire du Judaïsme - Paris, décembre 2009.

Bali R., 2010. Le dilemme identitaire des Juifs dans les premières années de la République turque. In Itinéraires sépharades. Complexité et diversité, Benbassa E. (dir.), PUPS, Paris, p. 127-140.

Behar D., 2006. L'inscription de l'héritier dans la lignée. Filiation, affiliation et réussite sociale en grande bourgeoisie turque. European Journal of Turkish Studies, $\mathrm{n}^{\circ} 4$.

Brink-Danan M., 2005. Reference points: text, context and change of definitions of turkish-jewish identity.Thèse de doctorat (Ph.D.), Palo Alto Californie, Stanford University, 236p.

Brink-Danan M., 2010. Exposer la tolérance. La différence et le doute dans un musée en Turquie. In Itinéraires sépharades. Complexité et diversité, Benbassa E. (dir.), PUPS, Paris, p. 141-155.

Choay F. 2009. Le patrimoine en questions. Paris, Seuil.

Debarre S., 2004. Alkent, une gated community alaturka? Conditions d'application d'un modèle américain en Turquie. Mémoire de maîtrise en géographie, université Lyon 2, juin 2004.

Galante A., 1985. Histoire des Juifs de Turquie. Istanbul, Isis Press, 9 volumes, 3040 p.

Güleryüz N., 2008. The synagogues of Istanbul. Istanbul, Gözlem, 209 p.

Güleryüz N., 2009. The turkish Jews, 700 years of togetherness. Istanbul, Gözlem, 64 p.

Karmi I. 1992. Jewish sites of Istanbul. Istanbul, Isis Press, 102 p. 
Kastoryano R., 1992a. Du Millet à la communauté : les Juifs de Turquie. Pardès, vol. 15, 1992, p. $137-158$.

Kastoryano R., 1992b. Trajectoires dans la ville : les Juifs à Istanbul. Critique, Août-Septembre 1992, vol. 543-544, p. 657-670.

Kastoryano R., 1992c. L'intégration politique par l'extérieur. La communauté juive de Turquie. Revue française de science politique, $42^{\mathrm{e}}$ année, $\mathrm{n}^{\circ}$ 5, p. 786-801.

Köksal A., 2010. Petite histoire de l'architecture stambouliote. Urbanisme, $\mathrm{n}^{\circ} 374$, septembreoctobre 2010, p. 56-59.

Levi M., 2011. Istanbul était un conte. Paris, Sabine Wespieser Editeur, 703 p.

Levy A., 2002. Jews, Turks, Ottomans. A shared history, fifteenth through the twentieth century. Syracuse University Press, New York, $304 \mathrm{p}$.

Marcilhacy D., 2005. Les Juifs sépharades de Turquie, une minorité sous presse. Outre-Terre, $\mathrm{n}^{\circ}$ 10, p. 379-386.

Mallet L. 2008. La Turquie, les Turcs et les Juifs.Histoire, Représentations, Discours et Stratégies. Istanbul, Isis Press, 616 p.

Mills A., 2010. Streets of memory. Landscape, tolerance, and national identity in Istanbul. Athens, Georgia, The University of Georgia Press, 288 p.

Morvan Y., Montabone B., 2010. Le pont de la rente. Les enjeux fonciers du troisième pont sur le Bosphore à Istanbul. Etudes foncières, $n^{\circ}$ 148, novembre-décembre 2010, p. 20-25.

Leyla Neyzi. Strong as Steel, Fragile as a Rose: A Turkish Jewish Witness to theTwentieth Century; Jewish Social Studies $12, \mathrm{n}^{\circ} 1$, p. 167-189.

Öktem K., 2008. Synagogue. In Becoming Istanbul, Istanbul, Garanti Platform, p. 308-309.

Pérouse J.-F., 2004. Les “cités sécurisées” des territoires périphériques de l'arrondissement d'Eyüp (Istanbul) ou les mirages de la distinction. L'information géographique, n² 2, p. 139-154.

Pérouse J.-F., 2005. Les non musulmans à Istanbul aujourd'hui : une présence en creux ? Le cas de l'arrondissement de Fatih. Revue des mondes musulmans et de la Méditerranée, n 107-110 : Identités confessionnelles et espace urbain en terres d'Islam, p. 261-295.

Pérouse J.-F., 2010. Istanbul depuis 1923 : la difficile entrée dans le XX ${ }^{\mathrm{e}}$ siècle ? In Istanbul. Histoire, promenades, anthologie \& dictionnaire, Monceau, N. (dir.). Paris, Robert Laffont, p. 231-290.

Romero R. 2008. Structural consequences of language shift: judeo-spanish in Istanbul. Thèse de doctorat (Ph.D.), Washington DC, Georgetown University, 175 p.

şeni N., 1990. Si je t'oublie Istanbul. Film documentaire, $30 \mathrm{~min}$, Istanbul Film Agency production. Servantie A., 2010. " Cimetières juifs », « Juifs », « Synagogues ». In Istanbul. Histoire, promenades, anthologie \& dictionnaire, Monceau, N. (dir.). Paris, Robert Laffont.

Shaw S., 1991. Jews of the Ottoman empire and the Turkish Republic. London, Macmillan.

Türkmen A. 2007.Galata Kulesi Sokak No. 23. Film documentaire.

Varol M.-C., 1989. Balat, faubourg juif d'Istanbul. Istanbul, Isis Press, 68 p. 


\section{NOTES}

1. Une synagogue est inaugurée à Büyükada en 1904, une autre à Heybeliada en 1956, où environ 250 familles séjournent alors, ainsi qu'une dernière à Burgazada en 1968, lieu de séjour pour plus de 360 familles cet été là (Güleryüz, 2008) ; en outre, plusieurs clubs, dont l'un exclusivement juif à Büyükada, font la joie des estivants.

2. Preuve comme bien souvent de ce chant du cygne, un musée des Iles a ouvert ses portes au cours de l'été 2010, sous l'égide d'Istanbul 2010, Capitale européenne de la culture.

3. Trustant plus de $50 \%$ du marché turc de la sécurité selon le quotidien Hürriyet Daily News (24 février 2011), Pronet est à présent divisé en deux branches : d'une part les systèmes d'alarmes et de l'autre les agents de sécurité, cette dernière étant dirigée par une personne influente au sein des instances communautaires.

4. C'est d'abord la bourgeoisie ashkénaze qui a fréquentée Burgazada dès les années 1950, et l'̂le est aujourd'hui moins sujette aux foules qui « envahissent » Büyükada.

5. Une agence immobilière ( City side»), un espace pour le «Pilates», une galerie d'art, un centre de cours de langue et au rez-de-chaussée, 2 cafés dont un Starbucks ainsi qu'une une succursale d'ING Bank.

6. La présence juive quotidienne à Kuzguncuk se limite à quelques anciens, dont un autiste bientôt octogénaire à la mémoire faramineuse qui sillonne l'artère principale, comme recherchant en vain de part et d'autre ses anciens voisins juifs. Son regard tombe parfois sur les deux copains de jeunesse, Niso et Aaron. Jeunes retraités, ceux-ci s'emploient à faire vivre la synagogue et viennent chaque jour tenir leurs heures de bureau, y boire le thé et recevoir les généreux dons des fidèles pour organiser les conviviales collations du samedi matin ou les menus travaux annuels de réfection et d'embellissement.

7. La communauté juive d'Izmir compte aujourd'hui moins de 1500 personnes et quelques familles vivent toujours à Bursa, Adana, Hatay, Ankara ou en Thrace (Güleryüz, 2009).

8. Rifat Bali relate l'exemple des prises de position de Munis Tekinalp, de son ancien nom Moise Cohen, en faveur de l'assimilation totale pensant qu'avec elle les Turcs se montreraient reconnaissants envers les Juifs de Turquie, ce qui ne fut pas le cas (Bali, 2010).

9. Classes moyennes supérieures laïcisées et au mode de vie occidental, en turc «beyaz türkler », dont Bağdat Caddesi est l'un des parangons.

10. Le cas de Kemerburgaz est un exemple de la complexité des dynamiques sociales stambouliotes. Vantant les attraits de cette « petite Amérique au cœur d'Istanbul », le quotidien proche du pouvoir Today's Zaman (19 décembre 2010), indiquait qu'entre autres célébrités, le très anti-israélien ministre des Affaires étrangères Ahmet Davutoğlu y avait élu domicile, rejoint dans cette enclave pour riches par le ministre de la Sécurité sociale, Ömer Dinçer.

11. La synagogue Kasturiya a été abandonnée dès 1937 puis démolie en 1992. L'ancien faubourg juif de Balat, dont Marie-Christine Varol a dressé le portrait urbain (Varol, 1989), illustre parfaitement les difficultés à préserver un patrimoine historique, excessivement coûteux à entretenir alors que les Juifs ont déserté le quartier depuis plusieurs générations et que les projets successifs de rénovation urbaine n'ont pas encore fait totalement flamber les prix. Ainsi, des inquiétudes pèsent sur la charmante synagogue en bois Istipol, pourtant protégée par l'équivalent des monuments historiques depuis 1987, sans compter l'hôpital, l'institution juive la plus vivante de ce secteur urbain, menacé de fermeture à court terme selon certaines sources. 


\section{RÉSUMÉS}

Les Juifs sont présents à Istanbul depuis plus de sept siècles. La communauté aux origines et localisations assez homogènes à partir de la période ottomane tend à se dissoudre et à se complexifier dans le courant du XXe siècle. Aujourd'hui, derrière l'optimisme de façade, la situation de la minorité juive devient de plus en plus préoccupante pour des raisons à la fois internes et externes : les attentats, la montée de l'antisémitisme ainsi que les contextes politique et géopolitique n'inspirent pas confiance, tandis que la cohésion communautaire s'affaiblit notamment du fait de l'étalement urbain. Via une enquête de terrain menée à partir des différentes synagogues (août 2009/avril 2011, plus d'une centaine de personnes interrogées), cet article se propose de revenir sur les multiples transformations ayant affecté la communauté juive d'Istanbul pour en envisager les perspectives spatiales et sociales.

Since more than seven centuries, Jews are in Istanbul. The origins and locations of the community, quite homogeneous from the time of Ottoman Empire, become more complex through twentieth century. Nowadays, behind an apparent optimism, the situation of the Jewish minority is more and more worrying because of internal and external reasons: attacks increase of anti-Semitism and political and geopolitical contexts damage the confidence; in the same time the community's cohesion is breaking down due to urban sprawl. Based on a fieldwork from the different synagogues of the city (august 2009/april 2011, with more than one hundred people interrogated) this paper retraces the urban trajectories of the Jewish community of Istanbul in order to show its spatial and social perspectives.

\section{INDEX}

Mots-clés : Istanbul, Juifs, patrimoine, synagogue, trajectoire résidentielle

Keywords : heritage, Istanbul, Jews, synagogue, urban trajectory

Thèmes : Sur le Champ - Sur le Terrain

\section{AUTEUR}

\section{YOANN MORVAN}

Yoann Morvan est en post doc, co-responsable de l'Observatoire Urbain d'Istanbul et chercheur associé de l'Institut Français d'Etudes Anatoliennes IFEA. yoannmorvan@yahoo.com 